\title{
ON THE MECHANISM OF INACTIVATION OF ANTIBODY ACTIVITY BY COBALT-60 GAMMA RADIATION-I*†
}

\author{
Shanti K. Seth $\ddagger$ and P. C. Rajam $\S$ \\ Department of Microbiology, School of Medicine, \\ The University of Michigan, Ann Arbor, Michigan
}

(Received 30 September 1965)

\begin{abstract}
Experimentally determined relationships between ${ }^{60} \mathrm{Co}$ radiation dose and inactivation of combining activity of anti-PAB indicated that a relatively large number of reactions were associated with inactivation. A progressive increase in gross molecular disorganization with increasing radiation dose was indicated by a change in viscosity. Some degree of molecular rearrangement was also suggested by an increased availability of an average of two to three disulphide bonds for reduction after irradiation in presence of urea. The molecular rearrangement suggested by viscosity and disulphide analysis is probably related to the inactivation process, rather than to mere secondary radiation-induced damage of material already inactivated, since, they were first measurable at doses of $7 \times 10^{4}$ and $15 \times 10^{4}$ rads, respectively, as compared to $D_{37}$ of $35 \times 10^{4}$ rads.

The irradiated material was found to contain, in addition to a grossly denatured fraction insoluble at low ionic strength, some subtly denatured fractions. These fractions were demonstrable by such sensitive techniques as elimination patterns in rabbits and rates of tryptic hydrolysis. Augmented inactivation above that resulting from irradiation alone, was demonstrated to follow mild post-irradiation heat treatment $\left(40-41^{\circ}\right.$ for $\left.3 \mathrm{hr}\right)$. This increased heat-induced inactivation was not accompanied by detectable changes in viscosity or in the number of disulphide bonds available for reduction. It appeared to relate to the presence of molecular species, in irradiated antibody solution, that had undergone almost complete disorganization of combining sites, since attempted stabilization of combining site by $p$-iodobenzoic added before heat treatment could not protect against the additional loss of activity. The degree of inactivation relating to the alternative possibility of free radical reactions with a critical group(s) in the combining site was evaluated by blocking experiments with specific haptens and was found to be insignificant.
\end{abstract}

\section{INTRODUCTION}

THE MEChANISM of inactivation of proteins by $\gamma$-radiation has been extensively studied and loss of biological activity has been variously ascribed to changes in the secondary and/or tertiary structure, or in the combining site of the molecule. When ovalbumin was irradiated with ${ }^{60} \mathrm{Co} \gamma$-radiation in the dry state, changes in optical rotation and solubility, accompanied with the formation of a number of molecular species with different antigenic properties, were observed.(1-3) After exposure of bovine serum albumin (BSA) to $10^{6}-10^{7}$ rads of $2 \mathrm{MeV}$ electrons, an increase in the number of disulphide bonds reducible with $\beta$-mercaptoethylamine was observed by Alexander and Hamilton. ${ }^{(4)}$ Decreased solubility of protein with increasing radiation dose was ascribed to aggregation and was correlated with an increase in the weight average molecular weight. Although $45 \mathrm{eV}$ was found to be sufficient to denature BSA appreciably ${ }^{(5)}$ only one molecule in five had a single

* Supported by grants from National Institutes of Health (C-3661, C3).

+ Work performed in partial fulfillment of the requirements for the degree of Doctor of Philosophy, The University of Michigan.

$\ddagger$ Present addresses: $\ddagger$ Department of Zoology, University of Texas, Austin, Texas; $\S$ Foundation for Research on the Nervous System, Boston, Massachusetts. 
cysteine residue and one in six had a histidine residue changed. Jackson ${ }^{(6)}$ studied the inactivation of anti-p-azobenzoate (anti-PAB), anti human serum albumin (anti-HSA) and anti bovine- $\gamma$-globulin (anti-BGG) in dilute aqueous solutions in terms of loss of, both precipitating and combining activity. Inactivation of activity was by an indirect process and loss of activity was independent of the concentration of specifically precipitable antibody in the preparation. Irradiation of anti HSA in the dose range of $20-45 \times 10^{4}$ rads resulted in progressive shift of equivalence zones and diminution of total precipitable antibody with increasing dose. Non-specific coprecipitation of irradiation products with undenatured antibody and increasing formation of rapidly sedimenting components $(9 S$ and $11 S)$ insoluble at low ionic strength was noted. Similar formation of $17 S-58 S$ components after irradiation of lyophilized human- $\gamma$-globulin has been observed by Sweet and Leone. (7) On the other hand Ray, Hutchinson and Morowitz ${ }^{(8)}$ observed a protective effect of the substrate when enzyme substrate complex was irradiated and Okada ${ }^{(9)}$ observed a correlation between the loss of enzyme activity and destruction by radiations of one out of a total of four tryptophan residues in the $D^{\prime}$ Nase molecule. The tryptophan destroyed was presumably in the active site.

In the present study the relative importance of non-specific structural degradation as compared to specific reaction of radiation with a group or groups in the active site has been examined. Anti-PAB $\gamma$-globulin amenable to close quantitation of activity loss by measurements of hapten binding, was employed. Evidence is presented that the inactivation of anti-PAB binding activity by radiation in dilute aqueous solutions, is due to a major degree of 'stepwise' changes in the secondary and tertiary structure of the molecule, concomitant with the formation of molecules with intermediate degrees of stability and activity. Inactivation due to radiation induced changes in the antibody combining site play a minor role.

\section{MATERIALS AND METHODS}

Immunizing antigens. (a) Bovine $\gamma$-globulin $p$-azobenzoate (PAB-BGG) was prepared by diazotizing $300 \mathrm{mg} p$-aminobenzoic acid (PAB) and coupling to $10 \mathrm{gm}$ BGG (Armour Fraction II) at $\mathrm{pH} 9-9.5$ and $0^{\circ}$. The antigen was dialysed against four 10-1. portions of saline borate buffer $\mathrm{pH} 8.0 \tau / 2=0.16$, at $4-5^{\circ}$ over a period of 5 days and then diluted to a concentration of $20 \mathrm{mg} / \mathrm{ml}$. Aliquots were stored frozen at $-20^{\circ}$. (b) trimethyl ( $p$-azophenyl) bovine $\gamma$-globulin, (TMAP-BGG), $557 \mathrm{mg}$ trimethyl $p$-aminophenylammonium chloride (TMAPCl) prepared according to Pinnow and Koch ${ }^{(10)}$ and Grossberg(11) was diazotized and coupled to $5 \mathrm{~g}$ BGG in saline-borate buffer. The coupled product precipitated on dialysis. The precipitate was made into a slurry, diluted to approximately $30 \mathrm{mg} / \mathrm{ml}$ in saline-borate buffer and aliquot stored at $-20^{\circ}$.

Test antigens. (a) Rabbit- $\gamma$-globulin $p$-azobenzoate (RGG-PAB), $30 \mathrm{mg}$ PAB was diazotized and coupled to $500 \mathrm{mg}$ ethanol fractionated rabbit- $\gamma$-globulin (RGG) obtained from serum pools from non-immunized rabbits. The antigen was further treated as described for BGG-PAB. (b) Trimethyl ( $p$-azophenyl) rabbit- $\gamma$-globulin (RGG-TMAP). Twenty milligrams RGG in $5 \mathrm{ml}$ saline-borate buffer, $\mathrm{pH} 8 \cdot 0$, were coupled to $11 \mathrm{mg}$ diazotized TMAPCl. The coupled product was dialysed and stored as above. 
Haptens. (a) Radioactive $p$-iodobenzoic acid (PIB-131I) was prepared by heatexchange with carrier free $\mathrm{Na}^{131} \mathrm{I}^{(12)}$ A solution with a specific activity of $50 \mathrm{mc} /$ $\mathrm{mM}$ was obtained. A working solution containing $4.03 \times 10^{6} \mathrm{M}$ PIB per $0.1 \mathrm{ml}$ and counting 5000 to 8000 counts/min was obtained by suitable volume dilutions of stock and addition of appropriate amounts of carrier PIB. Radioactivity was counted in a Nuclear Chicago well-type scintillation counter. (b) Radioactive (trimethyl-p-iodophenyl) ammonium chloride (TMIP-131I). TMIP was prepared and radio-labeled by heat-exchange with carrier free $\mathrm{Na}^{131} \mathrm{I}$ according to Grossberg. ${ }^{(11)}$ Five milligrams of TMIP were combined with $3 \mathrm{mC}{ }^{131} \mathrm{I}$ in pyrex ampule, the $\mathrm{pH}$ adjusted to 4.5 with methyl red, and the mixture heated in a Parr bomb at $150-160^{\circ}$ for $18 \mathrm{hr}$. The solution was purified by two successive precipitations with saturated $\mathrm{KI}$ and dissolution with water. The excess iodide was removed with $\mathrm{Ag}_{2} \mathrm{O}$. A preparation containing $50 \mathrm{mC} / \mathrm{mm}$ was obtained. Working solutions of TMIP_131I were obtained by suitable volume dilutions of stock and addition of appropriate amounts of carrier TMIP.

Antiserums. (a) Anti-p-azobenzoate (anti-PAB). New Zealand white rabbits of either sex received $0.5 \mathrm{ml}$ of $20 \mathrm{mg} / \mathrm{ml}$ PAB-BGG, intravenously, three times a week for a period of 10 weeks. Individual rabbit sera were tested with RGGPAB. ${ }^{13)}$ High titer sera were pooled, $\gamma$-globulin obtained by cold ethanol fractionation, (14) lyophilized and stored at $-20^{\circ}$. These preparations had a sedimentation coefficient of approximately $7 S$ in the analytical ultracentrifuge. The average percentage of protein specifically precipitable from anti-PAB was 4.5. (b) Antitrimethyl-( $p$-azophenyl) ammonium (anti-TMAP). Rabbits were injected with $30 \mathrm{mg}$ of a slurry of BGG-TMAP antigen, subcutaneously twice a week for a period of 4 weeks. ${ }^{(15)}$ The animals were trial bled 6 days after the last injection, and individual sera tested for precipitating activity with RGG-TMAP test antigen. Sera from high titer individuals were pooled and $\gamma$-globulin fractions separated and characterized as above. The average percentage of protein specifically precipitable was 5.

Equilibrium dialysis. This was performed in glass cells, consisting of two compartments separated by a semipermeable membrane as described by Carsten and Eisen. ${ }^{(16)}$ After equilibration for $48 \mathrm{hr}$ at $5.8 \pm 0.2^{\circ}$, approximately $0.4-0.5 \mathrm{ml}$ of solution from the protein compartment and the hapten compartment was transferred to previously weighed vials; the exact volume was calculated from the final weight and the specific gravity of the sample. Concentrations of PIB-131I on both sides of the semipermeable membrane were determined with a well-type scintillation counter at a counting efficiency of 36 per cent; a minimum of 12,800 counts (less than 1 per cent standard error) were recorded for each sample. Experiments were done in triplicate and comparable amounts of normal rabbit $\gamma$-globulin were utilized as controls on non-specific binding of hapten.

Irradiation of samples. The 5000 curie ${ }^{60} \mathrm{Co}$ source at the Phoenix Memorial Laboratory, University of Michigan, was used as the source of $\gamma$-radiation. All samples including controls were initially at a concentration of $10 \mathrm{mg} / \mathrm{ml}$ in salineborate buffer and were irradiated in glass vials that had been previously washed with chromic acid and thoroughly rinsed with distilled water and deionized water. The exact dose of radiation delivered was determined by the Fricke dosimeter according to Weiss. ${ }^{(17)}$ Dosimetry solutions were placed next to each sample, 
control protein and control dosimetry vials being held at the same temperature $\left(26^{\circ}\right)$.

Determination of protein concentration. Protein concentrations were measured by the Folin technique as modified by Lowry ${ }^{(18)}$ in all cases except those where viscosity of the solution was to be measured. Protein concentration in latter was determined gravimetrically. ${ }^{(19)}$

Reduction of $\gamma$-globulin. Reductions were carried out with $0.1 \mathrm{M} \beta$-mercaptoethylamine (2-MEA) at $26^{\circ}$ and $\mathrm{pH} 8.0$ for $2 \mathrm{hr}$. Urea twice recrystallized from $50 \%$ ethanol if used was at a final concentration of $8 \mathrm{M}$. Reduction mixtures were freed of excess 2 MEA through a column of IR-120 ( $\mathrm{H}^{+}$form) at $\mathrm{pH} 5 \cdot 0$. The number of $-\mathrm{SH}$ groups liberated were determined amperometrically. ${ }^{(20)}$

Viscosimetry. Viscosity measurements were performed in a Connon-Ubelhode semimicro dilution viscometer at $25 \cdot 1 \pm 0 \cdot 05^{\circ}$. The flow time for triple distilled deionized water in this viscometer was $211.68 \mathrm{sec}$. All viscosities reported are reduced kinematic viscosities (i.e. viscosity values were not multipled by solution density). Protein concentrations were $10 \mathrm{mg} / \mathrm{ml}$ in saline-borate buffer and were determined by gravimetric analysis. ${ }^{(19)}$

Rate of elimination of anti-PAB ${ }^{131} I$. (1) Anti-PAB was trace labeled with ${ }^{131} I$ by a slight modification of the micro-diffusion technique of Banerjee and Ekins. (21) A $25 \mathrm{ml}$ Erlenmeyer flask divided into two compartments by fusion of a centrally placed glass ring instead of Conway-dish was used. The labeled anti-PAB was freed of excess iodide and adsorbed iodine by passage through a $2 \times 15 \mathrm{~cm}$ column of Sephadex G-25 in $0.15 \mathrm{M} \mathrm{NaCl}$. A sample with specific activity of $15 \mu \mathrm{c} / \mathrm{mg}$ was obtained. (2) Measurement of rate of clearance: $8 \mathrm{mg}$ of anti-PAB ${ }^{131} \mathrm{I}$ in $2 \mathrm{ml}$ was injected into the marginal ear vein of each of three rabbits. After an interval of $15 \mathrm{~min}$, during which the injected protein equilibrated with tissues, $0.1 \mathrm{ml}$ blood was withdrawn from the ear artery of each rabbit. Later samples consisting of 0.1 to $0.5 \mathrm{ml}$ of blood were withdrawn at intervals of several hours, or each day for a maximum period of 12 days, after which all samples were diluted to $1 \mathrm{ml}$ and counted in a well-type scintillation counter. The per cent labeled protein remaining in blood at various intervals of time taking $15 \mathrm{~min}$ value, as 100 was then calculated. The plot of the geometric mean of the values from three rabbits, with calculated standard deviations, against time was graphically represented on a semilogarithmic scale.

Rate of tryptic hydrolysis of anti-PAB. Fifteen millilitres anti-PAB in salineborate buffer, at a concentration of $6.35 \mathrm{mg} / \mathrm{ml}$, were placed in a $250 \mathrm{ml}$ Erlenmeyer flask and $17 \mathrm{ml}$ of $0.1 \mathrm{M}$ borate-boric acid buffer, $\mathrm{pH} 8.0$ added. The flask was equilibrated in a water bath at $30^{\circ}$ for $30 \mathrm{~min}$, and $1 \mathrm{ml}, 0.3 \%$ aqueous trypsin (twice recrystallized, salt free, Worthington) added. The contents were mixed, $4 \mathrm{ml}$ of reaction mixture immediately withdrawn and added to $3 \mathrm{ml}, 10 \%$ trichloroacetic acid (TCA) in a $15 \mathrm{ml}$ centrifuge tube (zero time sample). Similar samples were withdrawn at appropriate time intervals over a $3-\mathrm{hr}$ period and added to TCA solution. The tubes were allowed to stand for $30 \mathrm{~min}$ at room temperature and centrifuged at room temperature $\left(25-26^{\circ}\right)$. Supernatant fluids were held at $5^{\circ}$. The rate of tryptic hydrolysis was measured by determination of absorption of supernatant fluids at $280 \mathrm{~m} \mu$, using zero time sample as control. The increase in optical density with time was plotted graphically. 
Protection of antibody combining sites with haptens. In a typical experiment $80 \mathrm{mg}$ of anti-PAB in $3 \mathrm{ml}$ saline-borate buffer, $\mathrm{pH} 8.0$ were mixed with $2.8 \times 10^{-5}$ moles hapten in saline-borate buffer and volume made up to $8 \mathrm{ml}$. The mixture was gently shaken at $25^{\circ}$ for $5 \mathrm{~min}$ and allowed to stand at $5^{\circ}$ overnight with occasional stirring. A $4 \mathrm{ml}$ portion of mixture was then exposed to the desired radiation dose and the rest used as a control on the effect of hapten on the binding activity without irradiation. Suitable irradiated and unirradiated anti-PAB solutions were included as controls. Following irradiation all samples were dialysed against water, followed by four 9-1. portions of saline-borate buffer for 5 days. The precipitate was centrifuged and the binding activity of the supernatant fluid measured by equilibrium dialysis.

\section{RESULTS}

Effect of increasing radiation dose on combining activity. A $1 \%$ solution of antiPAB in saline-borate buffer was exposed to doses from $0-33 \times 10^{4}$ rads. either at room temperature or at $0^{\circ}$. The irradiated solution was first dialysed against 3-1. cold distilled water and then against 3-1. portion of saline-borate buffer. The combining activity of the soluble fraction (a maximum of 15 per cent material became insoluble) was measured by dialysis to equilibrium against PIB-131I. Binding results representing per cent binding activity remaining at increasing radiation doses were plotted on a semilogarithmic scale (Fig. 1 curves I and III).

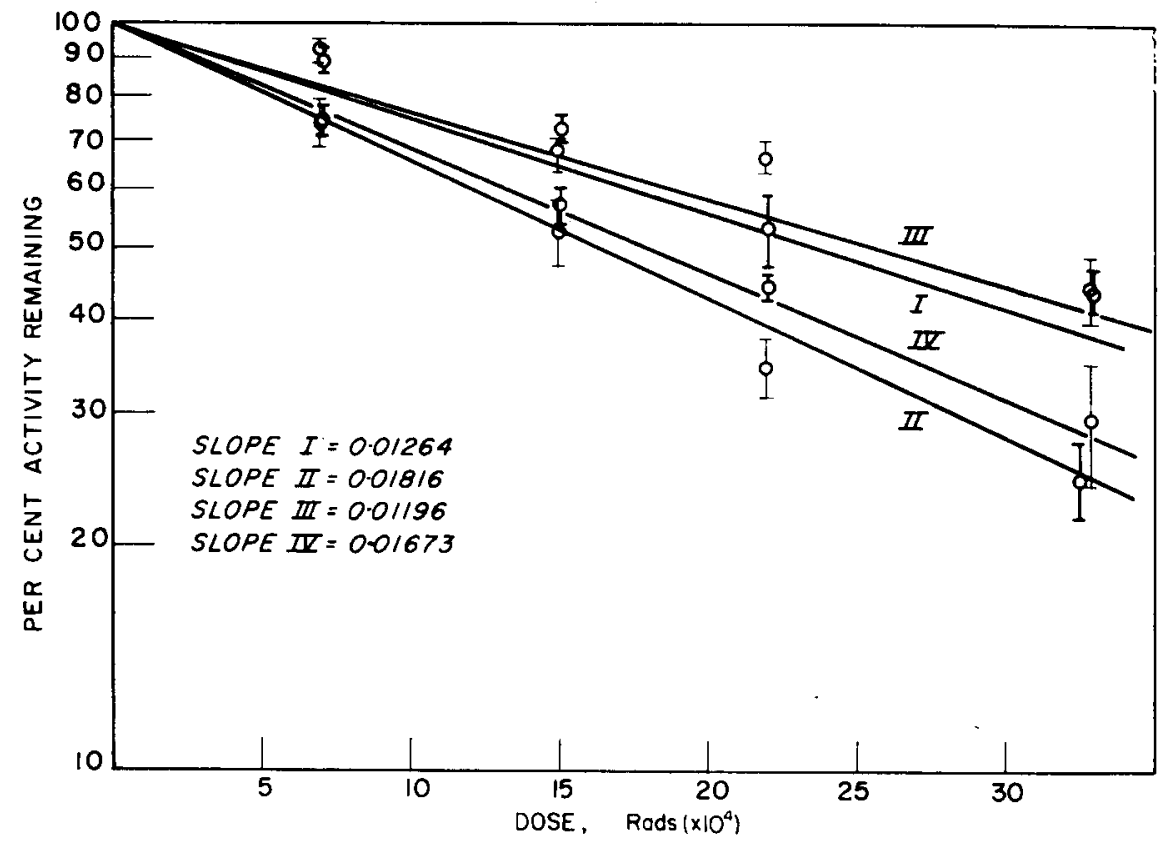

FIG. 1. Relation between increasing radiation dose, post-irradiation heat treatment and loss of anti-p-azobenzoate- $\gamma$-globulin combining activity. Lines drawn by the method of least squares. The points are average of triplicate determinations with mean deviations being indicated. I, anti-PAB irradiated at $0^{\circ}$. II, anti-PAB irradiated at $0^{\circ}$ followed by heating $\left(40-41^{\circ}\right.$ for $3 \mathrm{hr}$ ). III, anti-PAB irradiated at $26^{\circ}$. IV, anti-PAB irradiated at $26^{\circ}$ followed by heating ( $40-41^{\circ}$ for $3 \mathrm{hr}$ ). 


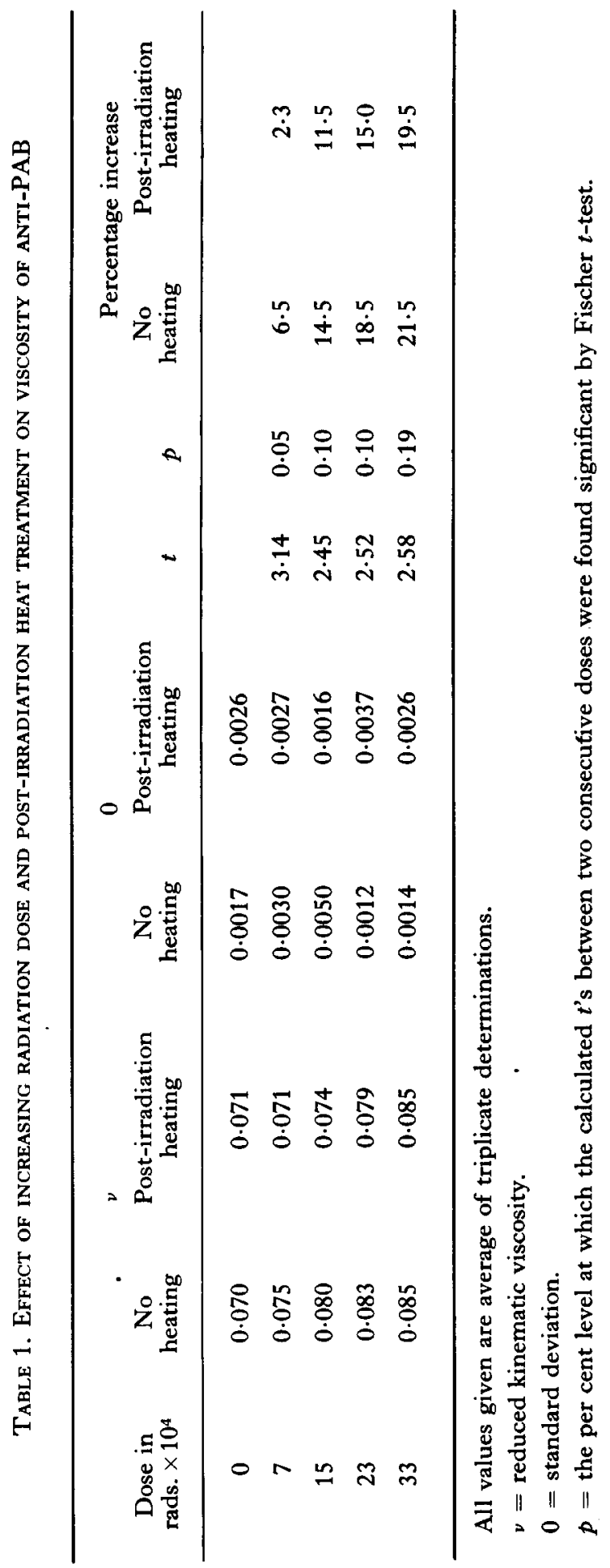


The exponential nature of this plot indicates that the radiochemical product formed by the interaction of antibody molecules with radiation reacts with free radicals as readily as the original material. Thus a dose which produces sufficient radicals to inactivate every molecule will bring about only 63 per cent inactivation since 37 per cent of the radicals will react with molecules that have already reacted once. From this graph $D_{37}$ (63 per cent inactivation) is $35 \times 10^{4}$ rads corresponding to a requirement of 20 ion pairs per molecule* $(G=0 \cdot 13)$. The net inactivation of combining activity, consistent with the observations of other workers using enzyme systems ${ }^{(22)}$ was independent of the temperature of irradiation, within the limits of temperature tested.

Effect of irradiation on viscosity. The viscosity of $1 \%$ solution of anti-PAB in saline-borate buffer, irradiated at $26^{\circ}$ in the range of $0-33 \times 10^{4}$ rads was measured at $25 \cdot 1 \pm 0 \cdot 05^{\circ}$. Changes in viscosity are presented in Table 1 . There was a small but progressive and statistically significant (Fischer $t$-Test) increase in viscosity with increasing dose. This increase, indicating a change in the conformation of the molecule, could have arisen from unfolding of the molecule, or due to aggregation resulting from inter- and intra-molecular reorganization. Although the viscosity data are insufficient to distinguish between unfolded and aggregated molecules, they do indicate a significant and progressive structural alteration.

TABLE 2. EFFECT OF INCREASING RADIATION DOSE AND POSTIRRADIATION HEAT TREATMENT ON REDUCIBLE DISULPHIDE BONDS OF ANTI-p-AZOBENZOATE

\begin{tabular}{cccc}
\hline & \multicolumn{3}{c}{ Moles-SH liberated per mole protein } \\
Dose in & in absence of urea & in $8 \mathrm{M}$ urea & in $8 \mathrm{M}$ urea \\
rads. $\times 10^{4}$ & $\tau / 2=0.16$ & $\tau / 2=0.16$ & $\tau 2 /=1.0$ \\
\hline 0 & $9.49 \pm 0.57$ & $24.53 \pm 0.37$ & $24.13 \pm 0.70$ \\
& $(8.59 \pm 0.70)$ & & \\
7 & $9.58 \pm 0 \cdot 50$ & $28.49 \pm 0.50$ & $27.15 \pm 0.70$ \\
& $(9.52 \pm 0.50)$ & & \\
15 & $9.58 \pm 0.70$ & $30.56 \pm 0.90$ & $30.10 \pm 0.10$ \\
& $(8.75 \pm 0 \cdot 70)$ & & \\
22 & $9.58 \pm 0.60$ & $29.70 \pm 0.20$ & $29.70 \pm 0.30$ \\
& $(9.85 \pm 0 \cdot 30)$ & & \\
33 & $9.99 \pm 0 \cdot 20$ & $33.10 \pm 1.0$ & $30.10 \pm 0.40$ \\
& $(9.12 \pm 0.50)$ & & \\
\hline
\end{tabular}

$a$ All values reported are averages of triplicate determinations, standard deviations being shown. Reductions were carried out at $\mathrm{pH} 8.0$ with $2 \mathrm{MEA}$. Values in parentheses were obtained when anti-PAB was heated to $40-41^{\circ}$ for $3 \mathrm{hr}$ following irradiation.

Effect of irradiation on reducible disulphide bonds. In general, there is an increase in the number of disulfide bonds available for reduction after denaturation. ${ }^{(23)} \mathrm{An}$ increase in the reducible disulfide content of BSA after exposure to $2 \mathrm{MeV}$ electrons was, therefore, used as a criterion for changes in the secondary and tertiary structure of the BSA molecule by Alexander and Hamilton. ${ }^{(4)}$ Similarly, as a criterion of molecular disorganization, anti-PAB after irradiation was reduced with $2 \mathrm{MEA}$ in the presence or absence of urea (Table 2). It was observed that

* Calculations are based on the assumption that $1 \mathrm{rad}$ of $\mathrm{X}$-rays produces $3.1 \times 10^{9} \mathrm{M}$ of ion pairs from water. 
exposure to radiation alone, $(9 \cdot 99-\mathrm{SH} /$ mole protein) did not increase the reducible disulfide content (in absence of urea) of anti-PAB over that observed in the native state $(9 \cdot 49-\mathrm{SH} /$ mole protein). However, seven additional disulfide bonds could be reduced in the presence of $8 \mathrm{M}$ urea $(24.53-\mathrm{SH} / \mathrm{mole})$ even without prior exposure to radiation. Post-irradiation reduction of disulfide bonds in the presence of urea resulted in an increased availability of 2-3 bonds $(30 \cdot 10-\mathrm{SH} / \mathrm{mole})$ which could not be prevented by increasing the ionic strength during irradiation. The fact that irradiation does not lead to an increase in the extent of reduction in the native $\gamma$-globulin but does result in an increase in the presence of urea suggests irradiation enhancement of denaturation effects due to urea. Disulfide interchange during irradiation ${ }^{(24)}$ might also lead to increased suscepitibility to reduction.

Effect of irradiation on the combining activity in presence and absence of blocking agents. In the following experiments, the role of critical groups in the combining site, for loss of anti-PAB binding activity by radiation, was examined by specific blocking of the combining site with hapten both before and after irradiation. Since haptenic moieties are destroyed at a significant rate a number of specific haptens and organic molecules of similar structure but different specificity and radiosensitivity were employed e.g. (1) salicylic acid and benzoic acid with high $k_{a}$ for combination with anti-PAB and known radiosensitivity (2) $\alpha$-naphthoic acid with known radioresistence and low $k_{a}$ for combination and (3) $\alpha$-naphthol known to be radio-resistant but without any specificity for combination with anti-PAB.

Benzoic acid and salicylic $\left(k_{a}=0.43\right)^{(25)}$ known to be good protectors against radiation induced changes in the physico-chemical properties of polymeric system $^{(26)}$ and for HSA in solution ${ }^{(27)}$ were used in the first experiment. Anti-PAB solutions in saline-borate buffer at a concentration of $10 \mathrm{mg} / \mathrm{ml}$ were irradiated at a fixed dose of $33 \times 10^{4}$ rads. in presence of $2.8 \times 10^{-5}$ moles of salicylic acid and benzoic acid in the concentration range $2.8 \times 10^{-5}$ to $2.8 \times 10^{-8}$ moles as described in detail under 'Methods'. Appropriate unirradiated and irradiated controls were included. It is evident (Table 3 ) that both salicylic acid and benzoic acid when used at a concentration of $2 \cdot 8 \times 10^{-5}$ moles were able to protect against about 60 per cent of the loss of combining activity. If, however, the concentration of benzoic acid was decreased by half, only 20 per cent protection against damage was observed. If the total protection against radiation-induced damage was due to a specific blocking of the combining site and if one assumes a constancy in the total amount of hapten degraded by radiation at either concentrations used, the ultimate value for protection should be independent of the concentration of benzoic acid within certain limits. The concentration dependence of net protection by benzoic acid, therefore, indicates involvement of hapten in some non-specific manner; possible radiation induced damage of, and scavenging of free radicals by the free hapten. In order to test this possibility $\alpha$-naphthoic acid, with lower $G$ value (number of molecules changed per $100 \mathrm{eV}$ of energy deposited) and lower $k_{a}$ for combination with anti-PAB than benzoic acid ${ }^{(28)}$ and $\alpha$-naphthol with a similar $G$ value as $\alpha$-naphthoic acid but with no specificity for combination with anti$\mathrm{PAB}$, were used.

Results of protection against radiation-induced loss of combining activity by $\alpha$-naphthol and $\alpha$-naphthoic acid are given in Table 4. $\alpha$-Naphthoic acid was as good a protector as salicylic acid or benzoic acid. Since it has a very low association 
Antibody Inactivation by Radiation

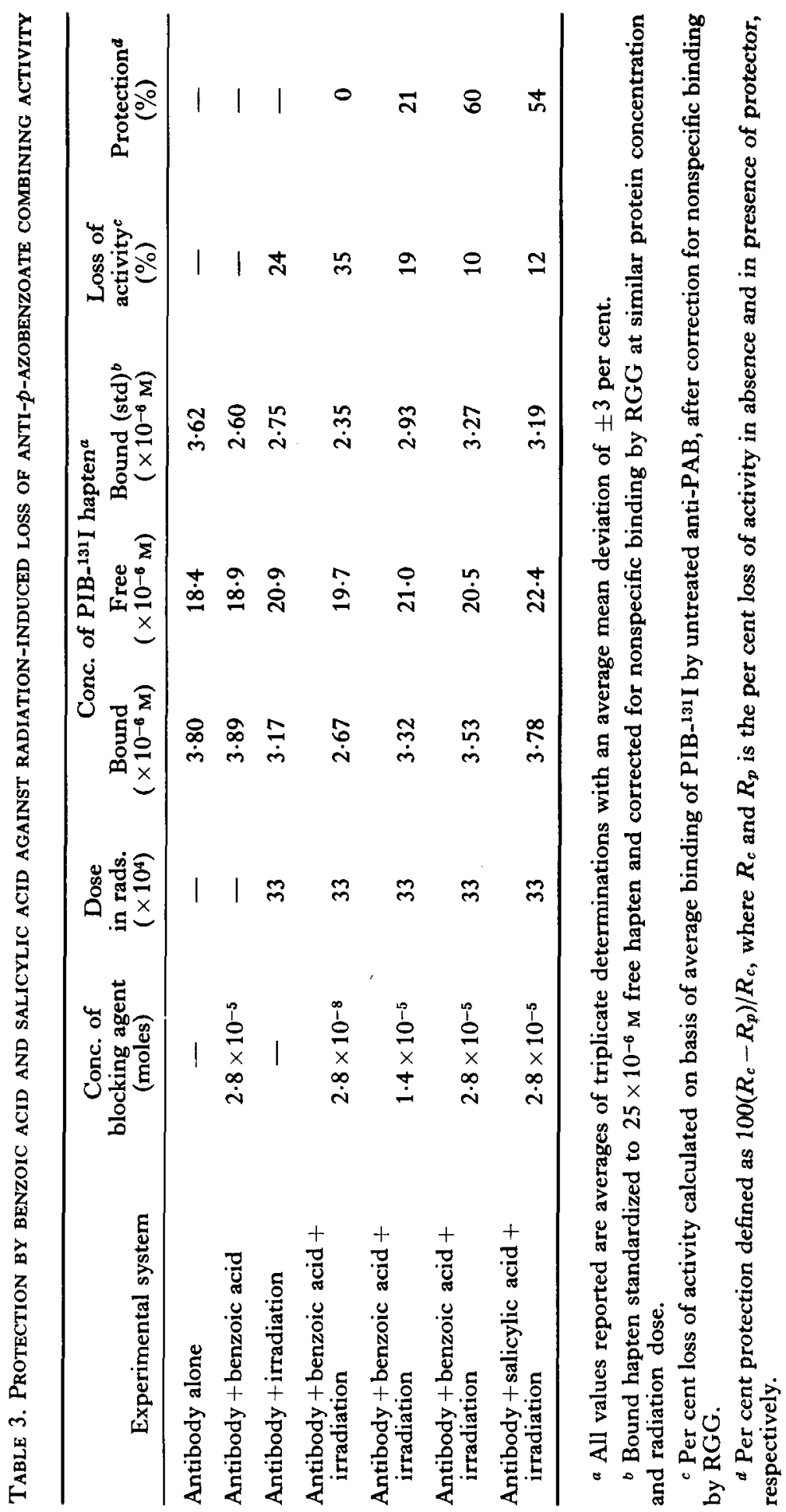


Shanti K. Seth and P. C. Rajam

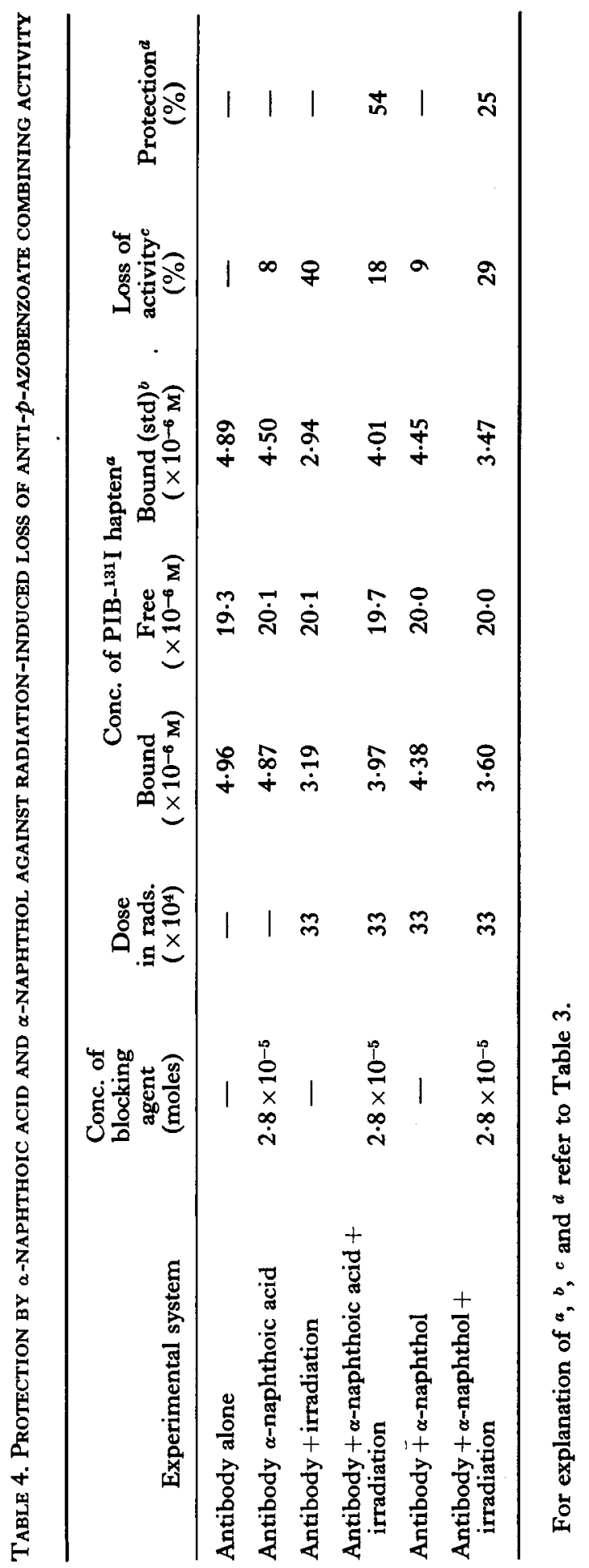


constant for combination with anti-PAB, a significant amount of added $\alpha$-naphthoic acid would have remained free in solution, thus the protection offered by $\alpha$ naphthoic acid may be mainly non-specific in nature. $\alpha$-Naphthol on the other hand offers only 25 per cent protection. Since $\alpha$-naphthol with $G$ value similar to $\alpha$-naphthoic acid does not bind at the combining site, at least 29 per cent (54-25) of the protection by $\alpha$-naphthoic acid could be of a specific nature. The specific protection by benzoic acid on anti-PAB was estimated by using anti-TMAP, an alternate antibody system bearing a negative charge in the combining site and thus unable to combine specifically with benzoic acid.(11)

The experiment was performed exactly as described under methods using the same concentration of benzoic acid $\left(2.8 \times 10^{-5}\right.$ moles $)$ and $1 \%$ anti-TMAP solution in saline-borate buffer. It is evident from Table 5 that benzoic acid was able to protect against 48 per cent of the loss of anti-TMAP activity. Thus it could be concluded that 12 per cent $(60-48)$ of the protective effect of benzoic acid on anti-PAB under comparable conditions of dose and concentration may be of a specific nature.

So far the effect of $60 \mathrm{Co} \gamma$-radiation on combining activity or physico-chemical properties of anti-PAB was studied separately. The progressive changes in viscosity and reducible disulfide bonds arising from continued random reactions of free radicals with anti-PAB molecule may give rise to a spectrum of molecular species with varying degrees of structural stability and activity. Augenstine et al. ${ }^{(29)}$ demonstrated the formation of molecular species with intermediate degree of activity during exposure of trypsin to ultraviolet irradiation by post-irradiation thermal treatment. Similarly, in the following experiments the presence of molecular species with different degrees of sensitivity has been demonstrated by use of mild heat treatment.

Effect of post-irradiation heat treatment on the combining activity and physicochemical properties of anti-PAB. Solutions of anti-PAB in saline-borate buffer, irradiated at doses ranging between $0-33 \times 10^{4}$ rads. were, following irradiation, heated at $40-41^{\circ}$ for $3 \mathrm{hr}$. The viscosity and total content of reducible disulfide bonds were measured as before. For the measurement of combining activity solutions were dialysed against cold distilled water and redialysed against salineborate buffer. The insoluble material was centrifuged and the binding activity of the supernatant solutions measured as described earlier. Data on residual combining activity following post-irradiation heat treatment is shown in Fig. 1 (Curves II and IV). A comparison of Curve I and Curve II shows that there was an additional 15 per cent loss of activity following heat treatment over that observed after irradiation alone. The increased loss of activity was found to occur to the same extent irrespective of the initial temperature of irradiation $\left(0^{\circ}-26^{\circ}\right)$. However, heat-augmentation of inactivation was not associated with further changes in viscosity (Table 1), or with total number of disulfide bonds available for reduction (Table 2), and thus is not characterized by major changes in the secondary and/or tertiary structure of the molecule.

Relation of augmented inactivation to stability of the combining site. Experiments were performed to determine the effect of stabilizing hapten ( $p$-iodobenzoic acid) present during post-irradiation heat treatment on the inactivation process. Changes in the combining activity loss and viscosity were measured. No protection by hapten against heat-augmented inactivation in either of the parameters was observed. 


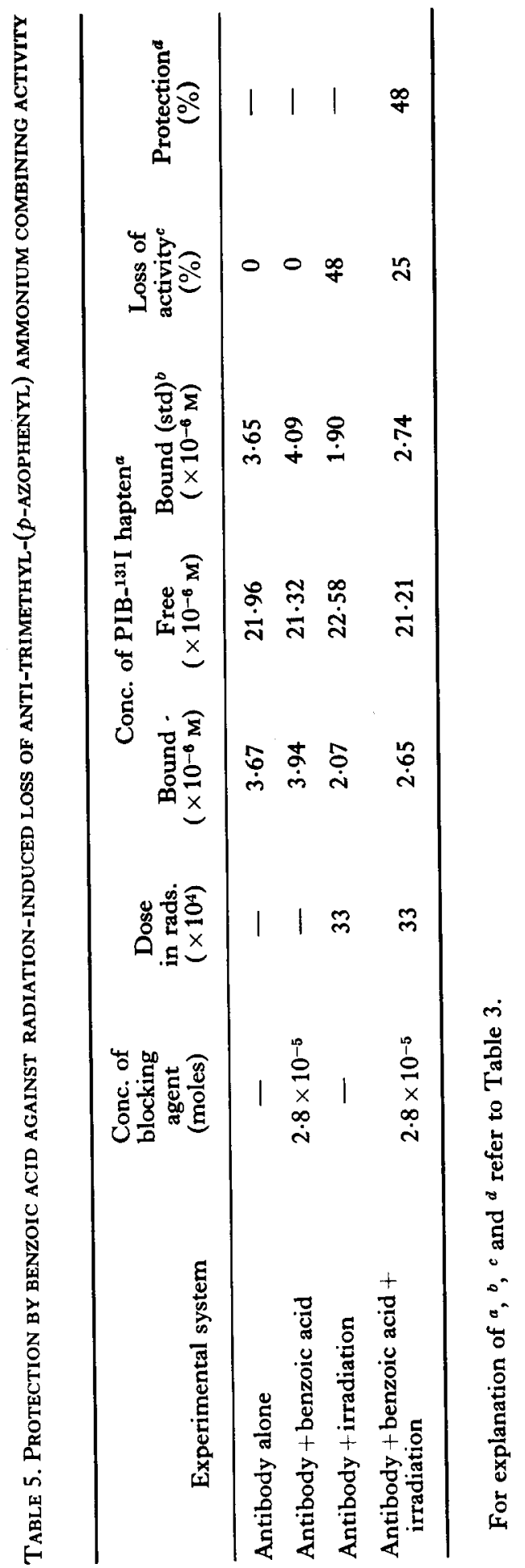


Effect of irradiation on the rate of elimination of anti-PAB-131I from the rabbit blood and on the kinetics of tryptic hydrolysis. The following experiments were performed to study the relations between molecular disorganizations and availability of combining sites for reaction, in the more subtly changed molecular species remaining soluble at low ionic strength, thus excluding a maximum of 15 per cent of grossly denatured material which became insoluble under these conditions. Using the rate of tryptic hydrolysis and the rate of elimination from rabbit blood, occurrence of denaturation in acetylated and guanidinated BSA molecules was shown by Maurer and Sri Ram;(30) this denaturation was not unequivocally demonstrable by physico-chemical techniques. Colson and Fredericq ${ }^{(31)}$ observed more active tryptic hydrolysis of BSA after exposure to ${ }^{60} \mathrm{Co} \gamma$-rays. Because of the extreme sensitivity of the method in revealing the presence of material in a subtly denatured state, this technique was used to analyse the physico-chemical status of molecules remaining in solution.

Parallel lots of anti-PAB at a concentration of $10 \mathrm{mg} / \mathrm{ml}$ in saline-borate buffer were exposed to $33 \times 10^{4}$ rads. of ${ }^{60} \mathrm{Co} \gamma$ rays (a) were the combining sites where blocked with $2 \cdot 8 \times 10^{-5}$ moles of benzoic acid and (b) where the combining sites were not protected with hapten. Protein solutions at the same concentration, unirradiated but in presence or absence of blocking hapten were used as controls. Following exhaustive dialysis against saline-borate buffer to remove the blocking hapten ${ }^{(32)}$ and against water to remove grossly denatured material, the samples were trace-labeled with ${ }^{131} \mathrm{I}$ and injected intravenously into each of three rabbits or diluted to a concentration of $6.35 \mathrm{mg} / \mathrm{ml}$ with $0.1 \mathrm{M}$-borate-boric acid buffer, $\mathrm{pH} 8 \cdot 1$ and hydrolysed with trypsin.

Elimination patterns are graphically represented in Fig. 2. Taking the amount of radioactivity remaining in blood at various intervals after injection of antiPAB-131I as a measure of the catabolic rate, it was observed (Fig. 2, Curve I) that about 20 per cent of the injected unirradiated anti-PAB-131I was present in the circulation after an interval of 5 days. This was also true of unirradiated haptentreated anti-PAB (Curve II). At the same time interval only 5 per cent anti-PAB exposed to a fixed dose of $33 \times 10^{4}$ rads in absence of blocking hapten (Curve IV) and $12 \%$ anti-PAB irradiated in presence of $2.8 \times 10^{-5}$ moles of benzoic acid (Curve III) remained in the blood stream. The rapid rate of elimination of antiPAB irradiated in the absence of hapten indicated the presence, in solution, of altered molecules. A significant degree of molecular disorganization, occurred even when the combining site was not available for reaction, although the extent of changes apparently was not as great as it was in the absence of blocking hapten. The results of tryptic hydrolysis are graphically represented in Fig. 3. Curve I representing the hydrolysis rate of the heat-denatured anti-PAB, showed it to be hydrolysed at a rate greater than the native molecules. The increased rate of tryptic hydrolysis of irradiated unblocked anti-PAB (Curve II) as compared to unirradiated anti-PAB (Curve IV) again indicated the presence of partially damaged molecules in solution. An intermediate rate of hydrolysis of anti-PAB irradiated in the presence of hapten (Curve III) showed that some molecular disorganization occurred irrespective of the presence of the specific hapten in solution during irradiation. These results are consistent with the formation of partially damaged molecules. 


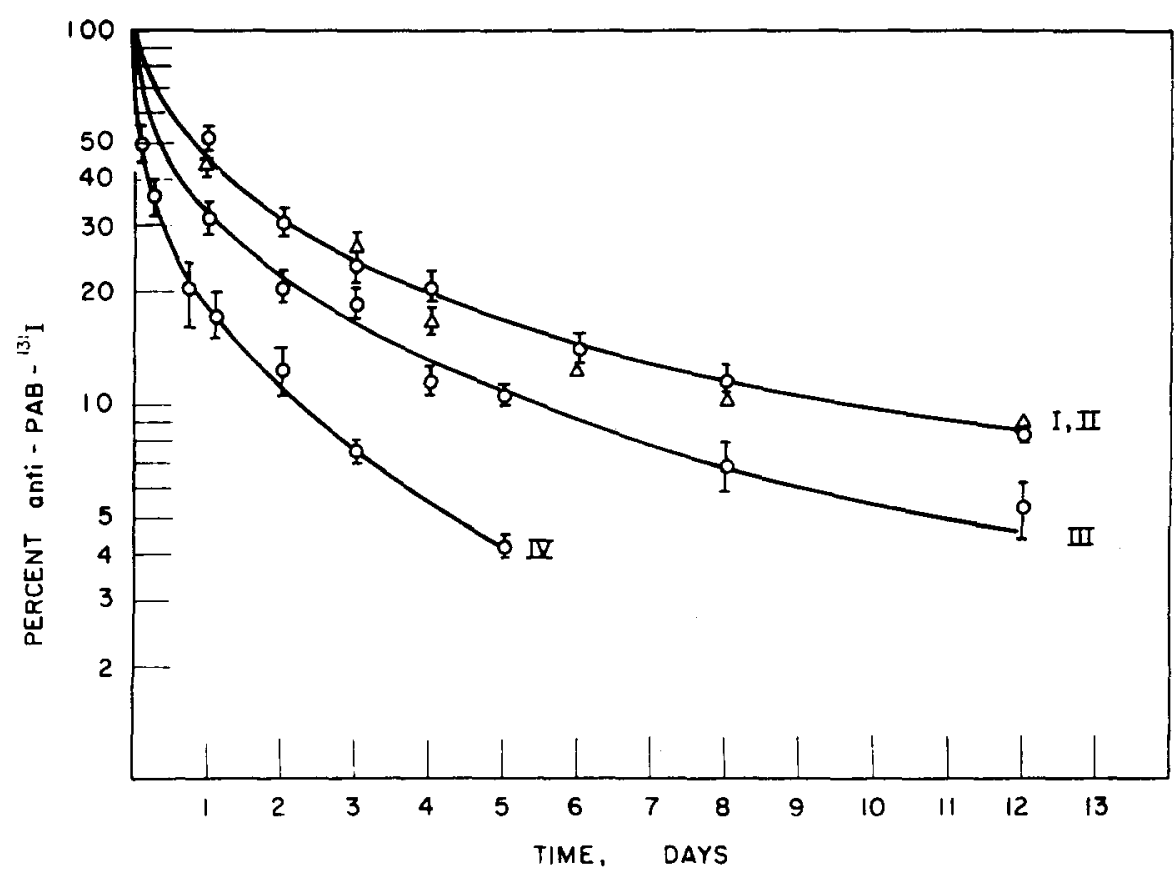

Fig. 2. Rate of elimination of anti-p-azobenzoate- $\gamma$-globulin (anti-PAB${ }^{131}$ I) from the rabbit. Points are averages of triplicate determinations, standard deviations being indicated. I, unirradiated anti-PAB (open circles); II, unirradiated anti-PAB treated with specific hapten (triangles). III, anti$P A B$ irradiated in presence of specific hapten. IV, anti-PAB irradiated in absence of specific hapten.

\section{DISCUSSION}

Jackson ${ }^{(6)}$ studied the effects of ${ }^{60} \mathrm{Co} \gamma$-radiation on anti-HSA, anti-PAB and anti-BGG under conditions similar to those described in this paper. Inactivation under these conditions was shown to be mediated by radiolysis products of water as evidenced by concentration dependence of inactivation at a fixed dose as well as by irradiation at $-76^{\circ}$ where free radical diffusion is greatly minimized. From the concentration-dependence curve a concentration of $10 \mathrm{mg} / \mathrm{ml}$ was found to preclude sufficient free radical recombination. Thus the average number of free radicals necessary to inactivate a single molecule of antibody could be computed with certainty. The percent inactivation in all the three systems studied (anti-HSA, from 10-40 per cent; anti-BGG from 15-26 per cent and anti-PAB from 4.5-23 per cent) was found independent of the concentration of antibody in the globulin preparation. Thus in the present work anti-PAB which contained only 4.5 per cent precipitable antibody was used.

The initial physico-chemical studies utilized irradiated material in toto, comprising both the material soluble and insoluble at low ionic strengths, nevertheless a small progressive and statistically significant increase in viscosity and increased availability of disulfide bonds for reduction at a dose $7 \times 10^{4}$ rads. and $15 \times 10^{4}$ rads., respectively, considerably below the 37 per cent dose seemed to bear a direct 
relationship to the processes leading to inactivation of combining activity. Determination of disulfide bonds as the net value of sulfhydryl groups obtained after reduction, in addition to any disulfide bonds possibly broken by radiation, did not show any increase over that observed in the native state. Even if radiation-induced scission of disulfide bonds occurred, the negative results observed could have been due to re-oxidation of free sulfhydryl groups. ${ }^{(33)}$ Following irradiation, reduction of disulfide bonds in presence of urea indicated the existence of two to three bonds not reducible under similar conditions in unirradiated anti-PAB. They may represent disulfide bridges re-formed due to oxidation of free formed sulfhydryl groups having different spatial distribution in a rearranged molecule. These bonds may also become manifest because of radiation induced 'generalized swelling' of the molecule resulting in increased solvent permeable volume. Urea and reducing agents would, therefore, be able to penetrate into regions that were inaccessible before irradiation. Such increased molecular solvent permeability arising from electrostatic repulsion of segments of the polypeptide chain, has been shown to be reversible at high ionic strengths for several globular proteins. ${ }^{(34)}$ The persistence of increased availability of these two to three bonds even when anti-PAB was irradiated in high ionic strength solvent suggest that these bonds may not be of the same characteristics as those already present in the native molecule.

In order to distinguish between gross molecular change and finer degrees of alternation and changes related with the entire population of molecules to antibody molecules the status of molecules present in solution after irradiation, and postirradiation heat treatment from which grossly denatured water-insoluble material (maximum 15 per cent) had been removed, was examined in terms of activity and physico-chemical properties. The rate of clearance from peripheral circulation of the homologous animal and the rate of tryptic hydrolysis of irradiated waterdialysed material were utilized for differentiation between relatively low degrees of molecular disorganization and overt molecular disorganization. This material was eliminated from the peripheral blood of the rabbit at a much faster rate than unirradiated (water-dialysed) control material (Fig. 2), indicating presence of altered molecular species. Similar results, confirming the presence of altered molecular species in water-dialysed material, were obtained from studies on rates of tryptic hydrolysis (Fig. 3). The presence of species with intermediate degrees of activity in the heterogeneous mixture of antibody and non antibody globulin molecules was demonstrated by mild post-irradiation heat treatment. This augmentation of inactivation and formation of products with decreased activity, was observed throughout the dose range studied $\left(0-33 \times 10^{4}\right.$ rads.) irrespective of the initial temperature of irradiation $\left(0^{\circ}\right.$ or $\left.26^{\circ}\right)$. A relationship between increased inactivation by post-irradiation heat treatment, and further changes in the secondary and tertiary structure of the molecule in addition to those observed by irradiation alone, was sought by measurement of viscosity (Table 1 ) and reducible disulfide bonds at this stage (Table 2). Absence of change in either of the properties over that observed after irradiation alone indicated that heat augmentation of inactivation could not be associated with major alteration in the secondary and/or tertiary structure. Thus in the stepwise process of molecular disorganization, molecules possessing partial activity could not be too far behind that terminal state of molecular disorganization corresponding to complete inactivation of 
antibody activity. It must be recognized that the progressive changes as measured by viscosity, reducible disulfide bonds and rate of digestion with trypsin are reflection of the changes in the entire population of antibody and non-antibody globulin molecules (nonspecific) whereas changes in combining activity and heat augmented loss of combining activity are the specific properties of the antibody under study e.g. post irradiation heating affects combining activity but not viscosity.

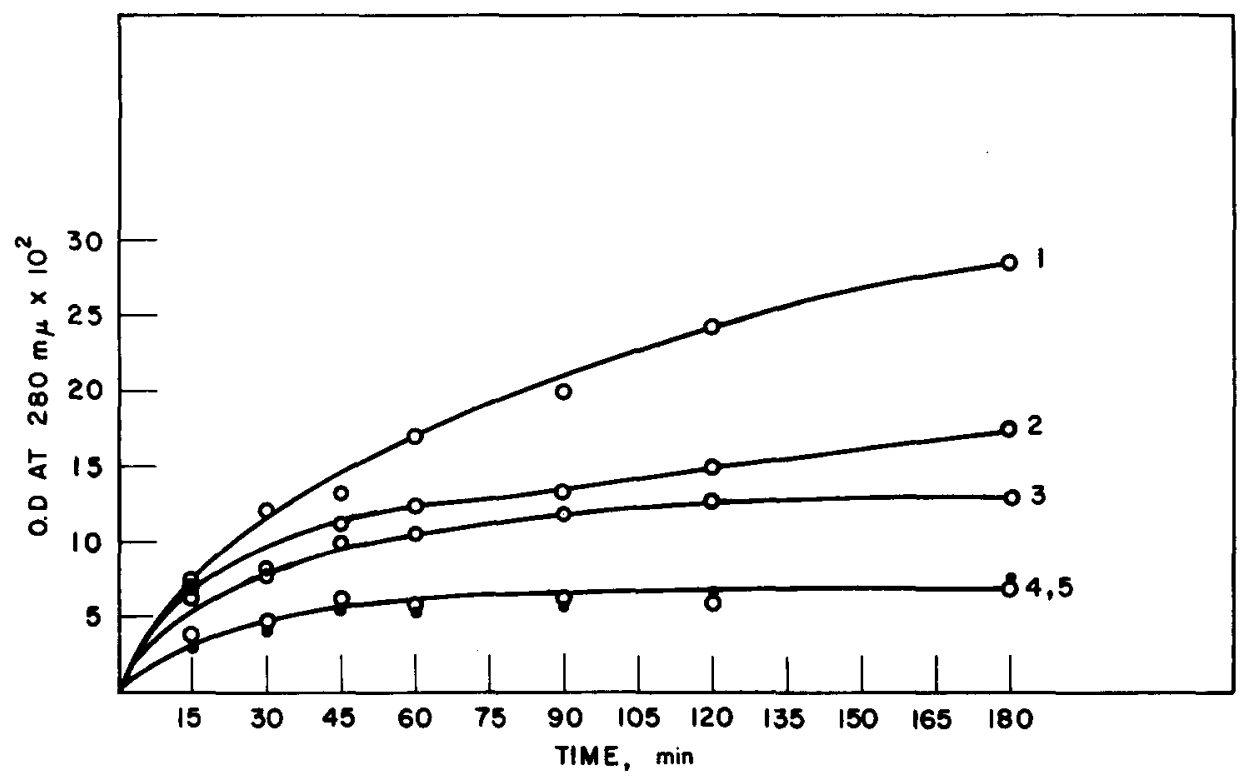

Fig. 3. Rates of tryptic hydrolysis of anti-p-azobenzoate- $\gamma$-globulin. Points represent an average of triplicate determinations. $I$, heat denatured anti$P A B$. II, anti-PAB irradiated at $33 \times 10^{4}$ rads. III, anti-PAB irradiated at $33 \times 10^{4}$ rads. in presence of specific hapten. IV, anti-PAB treated with hapten, unirradiated (solid circles). V, anti-PAB unirradiated (open circles)

An approximate measure of the extent of changes in the 'partially active' molecules can be roughly computed from the rate of tryptic hydrolysis of watersoluble irradiated anti-PAB. If an arbitrary value of 100 per cent disorganization is assigned to anti-PAB molecules denatured to insolubility by heating then a comparison of hydrolysis rates of this material and of irradiated anti-PAB solution, 180 min after initiation of tryptic hydrolysis, indicates occurrence of about 40-50 per cent disorganization in the latter. Computed on a similar basis, the rate of hydrolysis at the same interval of time for anti-PAB irradiated in presence of benzoic acid indicates only about 25-30 per cent disorganization. A certain degree of protection by benzoic acid against radiation-induced damage is thus obvious. Similar results for existence of denatured molecules and for protection against molecular disorganization were observed, if assessed by the rate of elimination of anti-PAB-131I from the circulation of the rabbit (Fig. 2). Considering these two results alone, the possibility that some part of the apparent protection against radiation-induced damage may be specific, is worthy of note. 
The significance of specific inactivation of combining activity due to reactions in the combining site, as opposed to a stepwise process of molecular disorganization was examined by blocking the combining sites with specific haptens before irradiation. A number of homologous haptens e.g. salicylic acid, benzoic acid, $\alpha$-naphthoic acid and a compound of similar molecular structure e.g. $\alpha$-naphthol were found to protect against radiation-induced loss of activity. The final degree of protection observed varied from about 60 per cent for benzoic and salicylic acid to 25 per cent for $\alpha$-naphthol. While the protection afforded by $\alpha$-naphthoic acid and $\alpha$-naphthol could be largely explained by a nonspecific radical scavenging process, benzoic acid and salicylic acid may act as specific protectors because of their capacity to specifically block the combining site. Excess benzoic acid used as a protector in these experiments which was not bound at the combining site may in addition act as a non-specific free radical trap. Benzoic acid does not combine specifically with anti-TMAP antibody(11) and hence any protection offered by benzoic acid against radiation-induced loss of combining activity of anti-TMAP may be considered a measure of non-specific radical scavenging by benzoic acid. Thus benzoic acid at a concentration of $2.8 \times 10^{-5}$ moles was able to protect against approximately 48 per cent of the loss of anti-TMAP combining activity. Since benzoic acid combines specifically with anti-PAB and at the same concentration is able to protect against 60 per cent of the loss of combining activity, it may be argued that a reaction of free radicals with a critical group or groups in the combining site takes place approximately 12 per cent of the time and results in inactivation. This 12 per cent specific protection was found to be statistically insignificant at a confidence limit of 95 per cent $(t=2 \cdot 08, N=10)$.

\section{REFERENCES}

1 Fricke H., Leone C. A. and Landman W., Nature, Lond. 180, 1423 (1957).

2 Fricke H., Landman W., L.eone C. A. and Vincent J., F. phys. Chem. 63, 932 (1959),

3 Leone C. A., Landman W. and Fricke H., Int. Conf. peaceful Uses atom Energy 2, 22, 504 (1958).

4 Alexander P., Hamilton L. D. G. and Stacey K. A., Radiat. Res. 12, 510 (1960).

5 Alexander P., in Ionizing Radiations and Immune Processes (Edited by LeONe C.) p. 75. Gordon \& Breach Service, New York (1962).

6 Jackson A. L., Ph.D. Thesis (1963).

7 Sweet G. H. and Leone C. A., Fedn Proc. Fedn Am. Socs exp. Biol. 23, 246 (1964).

${ }^{8}$ Ray D. K., Hutchinson F. and Morowitz H. J., Nature, Lond. 186, 312 (1960).

${ }^{9}$ OKAdA S. and Fletcher G., Radiat. Res. 16, 646 (1962).

${ }_{10}$ Pinnow J. and Koch E., Ber. dt. chem. Ges. 30, 2860 (1897).

11 Grossberg A. L. and Pressman D., J. Am. chem. Soc. 82, 5478 (1960).

12 Blau M., Johnson A. and Pressman D., Int. F. appl. Radiat. Isotopes 3, 217 (1958).

13 Nisonoff A. and Pressman D., F. Immunol. 80, 417 (1958).

14 Deutsch H. F., Meth. med. Res. 1, 284 (1951).

15 Fleicher S., Hardin R. L., Horowitz J., Zimmerman M., Gresham E., Turner J. E., Burnett J. P. and Haurowitz F., Archs Biochem. Biophys. 92, 329 (1961).

16 CARSten M. and Eisen H., F. Am. chem. Soc. 77, 1273 (1955).

17 WeIss J., Nucleonics 10, 28 (1952).

${ }^{18}$ Lowry O. H., Rosebrough N. J., Fau A. L. and Randall R. S., J. biol. Chem. 193, 265 (1951).

19 Jergensons B., Archs. Biochem. Biophys. 71, 149 (1957).

20 Benesch R. E., Lardy H. A. and Benesch R., F. biol. Chem. 216, 663 (1955).

21 BanerjeE R. N. and Ekins R. P., Nature, Lond. 192, 746 (1961).

${ }^{22}$ SETLOw R. B., Proc. natn. Acad. Sci. U.S.A. 38, 166 (1952).

${ }^{23}$ MARkus G., Protides biol. Fluids 11, 37 (1964). 
24 Rosen. D., Biochem. F. 72, 597 (1959).

25 Nisonoff A. and Pressman D., F. Am. chem. Soc. 79, 1616 (1957).

26 Alexander P., Bace Z. M., Cousens S. F., Fox M., Harve A. and Lazar J., Radiat. Res. 2, 392 (1955).

27 Alexander P. and Toms D. J., Radiat. Res. 9, 509 (1958).

${ }^{28}$ Pressman D., Grossberg A. L., Pence L. H. and Pauling L., F. Am. chem. Soc. 66, 1731 (1944).

${ }^{29}$ Augenstine L. and Ghiron C., Proc. natn. Acad. Sci. U.S.A. 47, 1530 (1961).

30 Maurer P. H. and SRI Ram J., In Serological and Biochemical Comparisons of Proteins (Edited by CoLE W. H.) p. 56. Rutgers University Press (1958).

31 Colson C. and Frederice E., Bull. Soc. chim. Belg. 71, 492 (1962).

${ }^{32}$ Nisonoff A. and PRessman D., $\mathcal{F}$. Immunol. 83, 138 (1959).

33 Anfinsen C. B., In Symposium on Protein Structure (Edited by Neuberger A.) p. 222. Meuthen, London (1958).

34 TANFord C. B., In Symposium on Protein Structure (Edited by Neuberger A.) p. 35 Meuthen, London (1958). 